Sadulla Z. Jafarov

\title{
Approximation of functions by de la Vallée-Poussin sums in weighted Orlicz spaces
}

Received: 18 February 2016 / Accepted: 6 June 2016 / Published online: 27 June 2016

(C) The Author(s) 2016. This article is published with open access at Springerlink.com

Abstract We investigate problems of estimating the deviation of functions from their de la Vallée-Poussin sums in weighted Orlicz spaces $L_{M}(T, \omega)$ in terms of the best approximation $E_{n}(f)_{M, \omega}$.

Mathematics Subject Classification $30 \mathrm{E} 10 \cdot 41 \mathrm{~A} 10 \cdot 41 \mathrm{~A} 25 \cdot 46 \mathrm{E} 30$

$$
\begin{aligned}
& \text { الملخص }
\end{aligned}
$$

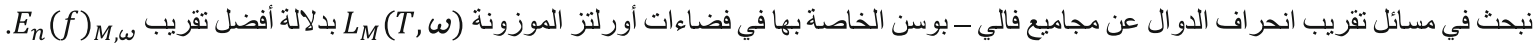

\section{Introduction, some auxiliary results and main results}

Let $M(u)$ be a continuous increasing convex function on $[0, \infty)$ such that $M(u) / u \rightarrow 0$ if $u \rightarrow 0$, and $M(u) / u \rightarrow \infty$ if $u \rightarrow \infty$. We denote by $N$ the complementary of $M$ in Young's sense, i.e., $N(u)=$ $\max \{u v-M(v): v \geq 0\}$ if $u \geq 0$. We will say that $M$ satisfies the $\Delta_{2}-$ condition if $M(2 u) \leq c M(u)$ for any $u \geq u_{0} \geq 0$ with some constant $c$, independent of $u$.

Let $\mathbb{T}$ denote the interval $[-\pi, \pi], \mathbb{C}$ the complex plane, and $L_{p}(\mathbb{T}), 1 \leq p \leq \infty$, the Lebesgue space of measurable complex-valued functions on $\mathbb{T}$.

For a given Young function $M$, let $\widetilde{L}_{M}(\mathbb{T})$ denote the set of all Lebesgue measurable functions $f: \mathbb{T} \rightarrow$ $\mathbb{C}$ for which

$$
\int_{\mathbb{T}} M(|f(x)|) \mathrm{d} x<\infty
$$

Let $N$ be the complementary Young function of $M$. It is well-known [28, p.69], [47, pp.52-68] that the linear span of $\widetilde{L}_{M}(\mathbb{T})$ equipped with the Orlicz norm

$$
\|f\|_{L_{M}(\mathbb{T})}:=\sup \left\{\int_{\mathbb{T}}|f(x) g(x)| \mathrm{d} x: g \in \widetilde{L}_{N}(\mathbb{T}), \int_{\mathbb{T}} N(|g(x)|) \mathrm{d} x \leq 1\right\},
$$

S. Z. Jafarov

Department of Mathematics, Faculty of Art and Science, Pamukkale University, 20070 Denizli, Turkey

S. Z. Jafarov $(\varangle)$

Mathematics and Mechanics Institute, Azerbaijan National Academy of Sciences, 9, B. Vahabzade St., Az-1141 Baku, Azerbaijan E-mail: sjafarov@pau.edu.tr 
or with the Luxemburg norm

$$
\|f\|_{L_{M}(\mathbb{T})}^{*}:=\inf \left\{k>0: \int_{\mathbb{T}} M\left(\frac{|f(x)|}{k}\right) \mathrm{d} x \leq 1\right\}
$$

becomes a Banach space. This space is denoted by $L_{M}(\mathbb{T})$ and is called an Orlicz space [28, p.26]. The Orlicz spaces are known as the generalizations of the Lebesgue spaces $L_{p}(\mathbb{T}), 1<p<\infty$. If $M(x)=M(x, p):=$ $x^{p}, 1<p<\infty$, then Orlicz spaces $L_{M}(\mathbb{T})$ coincide with the usual Lebesgue spaces $L_{p}(\mathbb{T}), 1<p<\infty$. Note that the Orlicz spaces play an important role in many areas such as applied mathematics, mechanics, regularity theory, fluid dynamics and statistical physics. Therefore, the investigation into the approximation of the functions by means of Fourier trigonometric series in Orlicz spaces is also important in these areas of research.

The Luxemburg norm is equivalent to the Orlicz norm as

$$
\|f\|_{L_{M}(\mathbb{T})}^{*} \leq\|f\|_{L_{M}(\mathbb{T})} \leq 2\|f\|_{L_{M}(\mathbb{T})}^{*}, \quad f \in L_{M}(\mathbb{T})
$$

holds true [28, p.80].

If we choose $M(u)=u^{p} / p, 1<p<\infty$, then the complementary function is $N(u)=u^{q} / q$ with $1 / p+1 / q=1$ and we have the relation

$$
p^{-1 / p}\|u\|_{L_{p}(\mathbb{T})}=\|u\|_{L_{M}(\mathbb{T})}^{*} \leq\|u\|_{L_{M}(\mathbb{T})} \leq q^{1 / q}\|u\|_{L_{p}(\mathbb{T})},
$$

where $\|u\|_{L_{p}(\mathbb{T})}=\left(\int_{\mathbb{T}}|u(x)|^{p} \mathrm{~d} x\right)^{1 / p}$ stands for the usual norm of the $L_{p}(\mathbb{T})$ space.

If $N$ is complementary to $M$ in Young's sense and $f \in L_{M}(\mathbb{T}), g \in L_{N}(\mathbb{T})$, then the so-called strong Hölder inequalities [28, p.80]

$$
\begin{aligned}
& \int_{\mathbb{T}}|f(x) g(x)| \mathrm{d} x \leq\|f\|_{L_{M}(\mathbb{T})}\|g\|_{L_{N}(\mathbb{T})}^{*}, \\
& \int_{\mathbb{T}}|f(x) g(x)| \mathrm{d} x \leq\|f\|_{L_{M}(\mathbb{T})}^{*}\|g\|_{L_{N}(\mathbb{T})}
\end{aligned}
$$

are satisfied.

The Orlicz space $L_{M}(\mathbb{T})$ is reflexive if and only if the $N$-function $M$ and its complementary function $N$ both satisfy the $\Delta_{2}-$ condition [47, p.113].

Let $M^{-1}:[0, \infty) \rightarrow[0, \infty)$ be the inverse function of the $N$-function $M$. The lower and upper indices $[4, \mathrm{p} .350]$

$$
\alpha_{M}:=\lim _{t \rightarrow+\infty}-\frac{\log h(t)}{\log t}, \quad \beta_{M}:=\lim _{t \rightarrow 0^{+}}-\frac{\log h(t)}{\log t}
$$

of the function

$$
h:(0, \infty) \rightarrow(0, \infty], \quad h(t):=\lim _{y \rightarrow \infty} \sup \frac{M^{-1}(y)}{M^{-1}(t y)}, \quad t>0
$$

first considered by Matuszewska and Orlicz [38], are called the Boyd indices of the Orlicz spaces $L_{M}(T)$.

It is known that the indices $\alpha_{M}$ and $\beta_{M}$ satisfy $0 \leq \alpha_{M} \leq \beta_{M} \leq 1, \alpha_{N}+\beta_{M}=1, \alpha_{M}+\beta_{N}=1$ and the space $L_{M}(\mathbb{T})$ is reflexive if and only if $0<\alpha_{M} \leq \beta_{M}<1$. The detailed information about the Boyd indices can be found in $[3,5-7,39]$.

A measurable function $\omega: \mathbb{T} \rightarrow[0, \infty]$ is called a weight function if the set $\omega^{-1}(\{0, \infty\})$ has Lebesgue measure zero. With any given weight $\omega$ we associate the $\omega$-weighted Orlicz space $L_{M}(\mathbb{T}, \omega)$ consisting of all measurable functions $f$ on $\mathbb{T}$ such that

$$
\|f\|_{L_{M}(\mathbb{T}, \omega)}:=\|f \omega\|_{L_{M}(\mathbb{T})} .
$$


Let $1<p<\infty, 1 / p+1 / p^{\prime}=1$ and let $\omega$ be a weight function on $\mathbb{T}$. $\omega$ is said to satisfy Muckenhoupt's $A_{p}$-condition on $\mathbb{T}$ if

$$
\sup _{J}\left(\frac{1}{|J|} \int_{J} \omega^{p}(t) \mathrm{d} t\right)^{1 / p}\left(\frac{1}{|J|} \int_{J} \omega^{-p^{\prime}}(t) \mathrm{d} t\right)^{1 / p^{\prime}}<\infty
$$

where $J$ is any subinterval of $\mathbb{T}$ and $|J|$ denotes its length.

Let us indicate by $A_{p}(\mathbb{T})$ the set of all weight functions satisfying Muckenhoupt's $A_{p}$-condition on $\mathbb{T}$.

According to [33,34, Lemma 3.3], and [34, Section 2.3] if $L_{M}(\mathbb{T})$ is reflexive and $\omega$ weight function satisfying the condition $\omega \in A_{1 / \alpha_{M}}(\mathbb{T}) \cap A_{1 / \beta_{M}}(\mathbb{T})$, then the space $L_{M}(\mathbb{T}, \omega)$ is also reflexive.

Let $L_{M}(\mathbb{T}, \omega)$ be a weighted Orlicz space, let $0<\alpha_{M} \leq \beta_{M}<1$ and let $\omega \in A_{\frac{1}{\alpha_{M}}}(\mathbb{T}) \cap A_{\frac{1}{\beta_{M}}}(\mathbb{T})$. For $f \in L_{M}(\mathbb{T}, \omega)$ we set

$$
\left(v_{h} f\right)(x):=\frac{1}{2 h} \int_{-h}^{h} f(x+t) \mathrm{d} t, 0<h<\pi, x \in T .
$$

By reference [18], Lemma 1.4, the shift operator $v_{h}$ is a bounded linear operator on $L_{M}(\mathbb{T}, \omega)$ :

$$
\left\|v_{h}(f)\right\|_{L_{M}(\mathbb{T}, \omega)} \leq c\|f\|_{L_{M}(\mathbb{T}, \omega)} .
$$

The function

$$
\Omega_{M, \omega}^{l}(\delta, f):=\sup _{\substack{0<h_{i} \leq \delta \\ 1 \leq i \leq l}}\left\|\prod_{i=1}^{l}\left(I-v_{h_{i}}\right) f\right\|_{L_{M}(\mathbb{T}, \omega)}, \quad \delta>0, \quad l=1,2, \ldots
$$

is called $k$-th modulus of smoothness of $f \in L_{M}(\mathbb{T}, \omega)$, where I is the identity operator.

It can easily be shown that $\Omega_{M, \omega}^{k}(\cdot, f)$ is a continuous, nonnegative and nondecreasing function satisfying the conditions

$$
\lim _{\delta \rightarrow 0} \Omega_{M, \omega}^{k}(\delta, f)=0, \quad \Omega_{M, \omega}^{k}(\delta, f+g) \leq \Omega_{M, \omega}^{k}(\delta, f)+\Omega_{M, \omega}^{k}(\delta, g)
$$

for $f, g \in L_{M}(\mathbb{T}, \omega)$.

The function conjugate to a $2 \pi$-periodic summable function on $[-\pi, \pi]$ given by

$$
\tilde{f}(x)=\lim _{\varepsilon \rightarrow+0}\left\{-\frac{1}{\pi} \int_{\varepsilon}^{\pi} \frac{f(x+t)-f(x-t)}{2 \tan \frac{t}{2}} \mathrm{~d} t\right\}=-\frac{1}{\pi} \int_{0}^{\pi} \frac{f(x+t)-f(x-t)}{2 \tan \frac{t}{2}} \mathrm{~d} t
$$

exists almost everywhere.

Let

$$
\frac{a_{0}}{2}+\sum_{k=1}^{\infty} A_{k}(x, f)
$$

be the Fourier series of the function $f \in L_{1}(\mathbb{T})$, where $A_{k}(x, f):=\left(a_{k}(f) \cos k x+b_{k}(f) \sin k x\right), k=$ $1,2, \ldots, a_{k}(f)$ and $b_{k}(f)$ are Fourier coefficients of the function $f \in L_{1}(\mathbb{T})$. For given $f \in L_{1}(\mathbb{T})$, let

$$
\tilde{f} \sim \sum_{k=1}^{\infty}\left(a_{k}(f) \sin k x-b_{k}(f) \cos k x\right)=\sum_{k=-\infty}^{\infty}\{-i \operatorname{signk}\} c_{k}(f) e^{i k x}
$$

be the conjugate Fourier series of $f$ with $c_{k}(f)=(1 / 2)\left(a_{k}(f)-i b_{k}(f)\right)$. It is known that the conjugate series to Fourier series $f \in L_{[0,2 \pi]}$ will not always be the Fourier series (see, e.g., [53, p.155]). 
The $n$-th partial sums, and de la Vallé e-Poussin sums [57] of series (1.1) are defined, respectively, as

$$
\begin{aligned}
S_{n}(x, f) & =\frac{a_{0}}{2}+\sum_{k=1}^{n} A_{k}(x, f), \\
V_{n, m}(x, f) & =\frac{1}{m+1} \sum_{\nu=n-m}^{n} S_{\nu}(x, f), \quad\left(0 \leq m \leq n, m, n \in Z_{+}:=\{1,2,3, \ldots\}\right) .
\end{aligned}
$$

The best approximation to $f \in L_{M}(\mathbb{T}, \omega)$ in the class $\prod_{n}$ of trigonometric polynomials of degree not exceeding $n$ is defined by

$$
E_{n}(f)_{M, \omega}:=\inf \left\{\left\|f-T_{n}\right\|_{L_{M}(\mathbb{T}, \omega)}: T_{n} \in \prod_{n}\right\} .
$$

Note that the existence of $T_{n}^{*} \in \Pi_{n}$ such that

$$
E_{n}(f)_{M, \omega}=\left\|f-T_{n}^{*}\right\|_{L_{M}(\mathbb{T}, \omega)}
$$

follows, for example, from Theorem 1.1 in [10, p.59].

Let $W_{M}^{r}(\mathbb{T}, \omega),(r=1,2, \ldots)$ be the class of functions such that $f^{(r-1)}$ is absolutely continuous and $f^{(r)} \in L_{M}(\mathbb{T}, \omega)$ becomes a Banach space under the consideration of the norm

$$
\|f\|_{W_{M}(\mathbb{T}, \omega)}:=\|f\|_{L_{M}(\mathbb{T}, \omega)}+\left\|f^{(r)}\right\|_{L_{M}(\mathbb{T}, \omega)} .
$$

Let $G$ be a finite domain in the complex plane $\mathbb{C}$, bounded by a rectifiable Jordan curve $\Gamma$, and let $G^{-}:=\operatorname{ext} \Gamma$. Further let

$$
T:=\{w \in \mathbb{C}:|w|=1\}, \quad D:=\operatorname{int} T \text { and } D^{-}:=\operatorname{ext} T .
$$

Let $w=\varphi(z)$ be the conformal mapping of $G^{-}$onto $D^{-}$normalized by

$$
\varphi(\infty)=\infty, \quad \lim _{z \rightarrow \infty} \frac{\varphi(z)}{z}>0,
$$

and $\psi$ stands for the inverse of $\varphi$.

Let $w=\varphi_{1}(z)$ indicate a function that maps the domain $G$ conformally onto the disk $|w|<1$. The inverse mapping of $\varphi_{1}$ will be shown by $\psi_{1}$. Let $\Gamma_{r}$ be the image of the circle $\left|\varphi_{1}(z)\right|=r, 0<r<1$ under the mapping $z=\psi_{1}(w)$.

Let us denote by $E_{p}$, where $p>0$, the class of all functions $f(z) \neq 0$ that are analytic in $G$ and have the property that the integral

$$
\int_{\Gamma_{r}}|f(z)|^{p}|\mathrm{~d} z|
$$

is uniformly bounded for $0<r<1$. We shall call the $E_{p}$-class the Smirnov class. If the function $f(z)$ belongs to $E_{p}$, then $f(z)$ has definite limiting values $f\left(z^{\prime}\right)$ almost every where on $\Gamma$, over all nontangential paths; $\left|f\left(z^{\prime}\right)\right|$ is summable on $\Gamma$; and

$$
\lim _{r \rightarrow 1} \int_{\Gamma_{r}}|f(z)|^{p}|\mathrm{~d} z|=\int_{\Gamma}\left|f\left(z^{\prime}\right)\right|^{p}\left|\mathrm{~d} z^{\prime}\right| .
$$

It is known that $\varphi^{\prime}=E_{1}\left(G^{-}\right)$and $\psi^{\prime} \in E_{1}\left(D^{-}\right)$. Note that the general information about Smirnov classes can be found in the books [12, pp.438-453], and [8, pp.168-185].

Let $L_{M}(\Gamma, \omega)$ be a weighted Orlicz space defined on $\Gamma$. We also define the $\omega$-weighted Smirnov-Orlicz class $E_{M}(G, \omega)$ as

$$
E_{M}(G, \omega):=\left\{f \in E_{1}(G): f \in L_{M}(\Gamma, \omega)\right\} .
$$

With every weight function $\omega$ on $\Gamma$, we associate another weight $\omega_{0}$ on $T$ defined by

$$
\omega_{0}(t):=\omega(\psi(t)), \quad t \in T .
$$


For $f \in L_{M}(\Gamma, \omega)$ we define the function

$$
f_{0}(t):=f(\psi(t)), \quad t \in T .
$$

Let $h$ be a continuous function on $[0,2 \pi]$. Its modulus of continuity is defined by

$$
\omega(t, h):=\sup \left\{\left|h\left(t_{1}\right)-h\left(t_{2}\right)\right|: t_{1}, t_{2} \in[0,2 \pi],\left|t_{1}-t_{2}\right| \leq t\right\}, \quad t \geq 0 .
$$

The curve $\Gamma$ is called Dini-smooth if it has a parameterization

$$
\Gamma: \varphi_{0}(s), \quad 0 \leq s \leq 2 \pi
$$

such that $\varphi_{0}^{\prime}(s)$ is Dini-continuous, i.e.,

$$
\int_{0}^{\pi} \frac{\omega\left(t, \varphi_{0}^{\prime}\right)}{t} \mathrm{~d} t<\infty
$$

and $\varphi_{0}^{\prime}(s) \neq 0[44, \mathrm{p} .48]$.

If $\Gamma$ is a Dini-smooth curve, then there exist [58] the constants $c_{1}$ and $c_{2}$ such that

$$
0 \leq c_{1} \leq\left|\psi^{\prime}(t)\right| \leq c_{2}<\infty, \quad|t|>1 .
$$

Note that if $\Gamma$ is a Dini-smooth curve, then by (1.2) we have $f_{0} \in L_{M}\left(\mathbb{T}, \omega_{0}\right)$ if $f \in L_{M}(\Gamma, \omega)$.

Let $1<p<\infty, \frac{1}{p}+\frac{1}{p^{\prime}}=1$ and let $\omega$ be a weight function on $\Gamma$. $\omega$ is said to satisfy Muckenhoupt's $A_{p}$-condition on $\Gamma$ if

$$
\sup _{z \in \Gamma r>0}\left(\frac{1}{r} \int_{\Gamma \cap D(z, r)}|\omega(\tau)|^{p}|\mathrm{~d} \tau|\right)^{1 / p}\left(\frac{1}{r} \int_{\Gamma \cap D(z, r)}[\omega(\tau)]^{-p^{\prime}}|\mathrm{d} \tau|\right)^{1 / p^{\prime}}<\infty,
$$

where $D(z, r)$ is an open disk with radius $r$ and centered $z$.

Let us denote by $A_{p}(\Gamma)$ the set of all weight functions satisfying Muckenhoupt's $A_{p}$-condition on $\Gamma$. For a detailed discussion of Muckenhoupt weights on curves (see, e.g., [4]).

Let $\Gamma$ be a rectifiable Jordan curve and $f \in L_{1}(\Gamma)$. Then, the function $f^{+}$defined by

$$
f^{+}(z):=\frac{1}{2 \pi i} \int_{\Gamma} \frac{f(s) d s}{s-z}, z \in G
$$

is analytic in G. Note that if $0<\alpha_{M} \leq \beta_{M}<1, \omega \in A_{1 / \alpha_{M}}(\Gamma) \cap A_{1 / \beta_{M}}(\Gamma)$ and $f \in L_{M}(\Gamma, \omega)$, then by Lemma 1.4 in [20] $f^{+} \in E_{M}(G, \omega)$.

Let $\varphi_{k}(z), k=0,1,2, \ldots$ be the Faber polynomials for $G$. The Faber polynomials $\varphi_{k}(z)$, associated with $G \cup \Gamma$, are defined through the expansion

$$
\frac{\psi^{\prime}(w)}{\psi(w)-z}=\sum_{k=0}^{\infty} \frac{\varphi_{k}(z)}{t^{k+1}}, \quad z \in G, \quad t \in D^{-}
$$

and the equalities

$$
\begin{gathered}
\varphi_{k}(z)=\frac{1}{2 \pi i} \int_{T} \frac{w^{k} \psi^{\prime}(w)}{\psi(w)-z} \mathrm{~d} w, \quad z \in G, \\
\varphi_{k}(z)=\varphi^{k}(z)+\frac{1}{2 \pi i} \int_{\Gamma} \frac{\varphi^{k}(s)}{s-z} \mathrm{~d} s, \quad z \in G^{-}, \quad k=0,1,2, \ldots
\end{gathered}
$$

hold [51, p.33-38].

Let $f \in E_{M}(G, \omega)$. Since $f \in E_{1}(G)$, we have

$$
f(z)=\frac{1}{2 \pi i} \int_{\Gamma} \frac{f(s) \mathrm{d} s}{s-z}=\frac{1}{2 \pi i} \int_{T} \frac{f(\psi(w)) \psi^{\prime}(w)}{\psi(w)-z} \mathrm{~d} w,
$$


for every $z \in G$. Considering this formula and expansion (1.3), we can associate with $f$ the Faber series

$$
f(z) \sim \sum_{k=0}^{\infty} a_{k}(f) \varphi_{k}(z), \quad z \in G,
$$

where

$$
a_{k}(f):=\frac{1}{2 \pi i} \int_{T} \frac{f(\psi(w))}{w^{k+1}} \mathrm{~d} w, \quad k=0,1,2, \ldots
$$

This series is called the Faber series expansion of $f$, and the coefficients $a_{k}(f), k=0,1,2, \ldots$ are said to be the Faber coefficients of $f$.

The $n$-th partial sums and de la Vallée-Poussin sums of the series (1.6) are defined, respectively, as

$$
\begin{aligned}
S_{n}(z, f) & =\sum_{k=o}^{n} a_{k}(f) \varphi_{k}(z), \\
V_{n, m}(z, f) & =\frac{1}{m+1} \sum_{\nu=n-m}^{n} S_{v}(z, f), \quad\left(0 \leq m \leq n, m, n \in Z_{+}\right) .
\end{aligned}
$$

Let $\Gamma$ be a Dini-smooth curve. Using the nontangential boundary values of $f_{0}^{+}$on $T$ we define the r-th modulus of smoothness of $f \in L_{M}(\Gamma, \omega)$ as

$$
\Omega_{\Gamma, M, \omega}^{l}(\delta, f):=\Omega_{M, \omega_{0}}^{l}\left(\delta, f_{0}^{+}\right), \quad \delta>0,
$$

for $l=1,2,3, \ldots$

Let $\mathcal{P}:=\{$ all polynomials (with no restriction on the degree) $\}$, and let $\mathcal{P}(D)$ be the set of traces of members of $\mathcal{P}$ on $D$. We define the operator $T$ as follows:

$$
\begin{aligned}
T & :=\mathcal{P}(D) \longrightarrow E_{M}(G, \omega), \\
T(P)(z) & :=\frac{1}{2 \pi i} \int_{T} \frac{P(w) \psi^{\prime}(w)}{\psi(w)-z} \mathrm{~d} t, \quad z \in G .
\end{aligned}
$$

Then, taking into account (1.4) and (1.5) we have

$$
T\left(\sum_{k=0}^{n} b_{k} w^{k}\right)=\sum_{k=0}^{n} b_{k} \varphi_{k}(z), \quad z \in G .
$$

We use the constants $c, c_{1}, c_{2}, \ldots$ (in general, different in different relations) which depend only on the quantities that are not important for the questions of interest

We need the following results.

Theorem 1.1 [18] Let $L_{M}(T, \omega)$ be a weighted Orlicz space with Boyd indices $0<\alpha_{M} \leq \beta_{M}<1$, and let $\omega \in A_{1 / \alpha_{M}}(T) \cap A_{1 / \beta_{M}}(T)$. Then for every

$f \in W_{M}^{r}(T, \omega)(r=0,1,2, \ldots)$ the inequality

$$
E_{n}(f)_{M, \omega} \leq \frac{c_{3}}{(n+1)^{r}} E_{n}\left(f^{(r)}\right)_{M, \omega}
$$

holds with a constant $c_{3}>0$ independent of $n$.

Theorem 1.2 [18] Let $L_{M}(T, \omega)$ be a weighted Orlicz space with Boyd indices $0<\alpha_{M} \leq \beta_{M}<1$, and let $\omega \in A_{1 / \alpha_{M}}(T) \cap A_{1 / \beta_{M}}(T)$. Then for every

$f \in L_{M}(T, \omega)$ the inequality

$$
E_{n}(f)_{M, \omega} \leq c_{4} \Omega_{M . \omega}^{l}\left(\frac{1}{n+1}, f\right)
$$

holds with a constant $c_{4}>0$ independent of $n$. 
Theorem 1.3 [26] Let $L_{M}(T, \omega)$ be a weighted Orlicz space with Boyd indices $0<\alpha_{M} \leq \beta_{M}<1$, and let $f \in L_{M}(T, \omega), \omega \in A_{1 / \alpha_{M}}(T) \cap A_{1 / \beta_{M}}(T)$. If the condition

$$
\sum_{n=1}^{\infty} n^{r-1} E_{n}(f)_{M, \omega .}<\infty
$$

is satisfied for $r \in Z_{+}$, then $\widetilde{f}^{(r)} \in L_{M}(T, \omega)$ and

$$
E_{n}\left(\widetilde{f}^{(r)}\right)_{M, \omega} \leq c_{5}\left\{(n+1)^{r} E_{n}(f)_{M, \omega .}+\left(\sum_{\mu=n+1}^{\infty} \mu^{r-1} E_{\mu}(f)_{M, \omega .}\right)\right\},
$$

where the constant $c_{5}$ is independent of $n$.

Lemma 1.4 Let $L_{M}(T, \omega)$ be a weighted Orlicz space with Boyd indices $0<\alpha_{M} \leq \beta_{M}<1$, and $\omega \in$ $A_{1 / \alpha_{M}}(T) \cap A_{1 / \beta_{M}}(T)$.

1. Then, for $r \in N$ and $f^{(r)} \in L_{M}(T, \omega)$ the estimate

$$
\left\|f^{(r)}\right\|_{L_{M}(\mathbb{T}, \omega)} \leq c_{6}\left\{n^{r}\|f\|_{L_{M}(\mathbb{T}, \omega)}+E_{n}\left(f^{(r)}\right)_{M, \omega .}\right\}
$$

holds with a constant $c_{6}>0$ independent of $n$.

2. If $\widetilde{f}^{(r)} \in L_{M}(T, \omega)$, then the estimate

$$
\left\|\widetilde{f}^{(r)}\right\|_{L_{M}(\mathbb{T}, \omega)} \leq c_{7}\left\{n^{r}\|f\|_{L_{M}(\mathbb{T}, \omega)}+E_{n}\left(\widetilde{f}^{(r)}\right)_{M, \omega .}\right\}
$$

holds with a constant $c_{7}>0$ independent of $n$.

Proof The function $f^{(r)}$ can be written in following form:

$$
f^{(r)}(x)=\left(f^{(r)}(x)-V_{2 n, n}\left(x, f^{(r)}\right)\right)+V_{2 n, n}\left(x, f^{(r)}\right) .
$$

Then, by (1.9) we obtain

$$
\left\|f^{(r)}\right\|_{L_{M}(\mathbb{T}, \omega)} \leq\left\|f^{(r)}-V_{2 n, n}\left(., f^{(r)}\right)\right\|_{L_{M}(\mathbb{T}, \omega)}+\left\|V_{2 n, n}\left(., f^{(r)}\right)\right\|_{L_{M}(\mathbb{T}, \omega)} .
$$

By [18] the following inequality holds:

$$
\left\|f-S_{n}(., f)\right\|_{L_{M}(\mathbb{T}, \omega)} \leq c_{8} E_{n}(f)_{M, \omega} .
$$

Then, from the inequality (1.11) we conclude that

$$
\left\|f^{(r)}-V_{2 n, n}\left(., f^{(r)}\right)\right\|_{L_{M}(\mathbb{T}, \omega)} \leq E_{n}\left(f^{(r)}\right)_{M, \omega} .
$$

Using the Bernstein inequality for weighted Orlicz spaces [18], we have

$$
\begin{aligned}
& \left\|V_{2 n, n}\left(., f^{(r)}\right)\right\|_{L_{M}(\mathbb{T}, \omega)}=\left\|\frac{\mathrm{d}^{r}}{\mathrm{~d} x^{r}} V_{2 n, n}(x, f)\right\|_{L_{M}(\mathbb{T}, \omega)} \\
& \quad \leq c_{9}(2 n)^{r}\left\|V_{2 n, n}(., f)\right\|_{L_{M}(\mathbb{T}, \omega)} \leq c_{10} n^{r}\|f\|_{L_{M}(\mathbb{T}, \omega)} .
\end{aligned}
$$

Now combining (1.10), (1.12) and last relation, we obtain the inequality (1.7) of Lemma 2.1. The inequality (1.8) is proved to be similar.

The proof of Lemma 1.4 is completed.

Theorem 1.5 [20] Let $\Gamma$ be a Dini-smooth curve and $L_{M}(\Gamma)$ be a reflexive Orlicz space. If $\omega \in A_{1 / \alpha_{M}}(\Gamma) \cap$ $A_{1 / \beta_{M}}(\Gamma)$, then the linear operator $T:=P(D) \longrightarrow E_{M}(G, \omega)$ is bounded. 
Theorem 1.6 [20] If $\Gamma$ is a Dini-smooth curve, $0<\alpha_{M} \leq \beta_{M}<1$, and $\omega \in A_{1 / \alpha_{M}}(\Gamma) \cap A_{1 / \beta_{M}}(\Gamma)$, then the operator

$$
T: E_{M}\left(D, \omega_{0}\right) \longrightarrow E_{M}(G, \omega)
$$

is one-to-one and onto.

The problems of approximation theory in weighted and nonweighted Lebesgue spaces, weighted and nonweighted Orlicz spaces have been investigated by several authors (see, e.g., [13-27,30-32,36,37,45,46]).

Note that the approximation problems by trigonometric polynomials in weighted Lebesgue spaces with weights belonging to the Muckenhoupt class $A_{p}(\mathbb{T})$ were studied in [13,36,37]. Detailed information on the weighted polynomial approximation can be found in the books $[9,41]$.

In the present paper, we investigate the problems of estimating the deviation of functions from their de la Vallée-Poussin sums in weighted Orlicz spaces $L_{M}(T, \omega)$.

This result is applied to estimate of approximation of de la Vallé e-Poussin sums of Faber series in weighted Smirnov-Orlicz classes defined on simply connected domains of the complex plane in terms of the modulus of smoothness. We also study the approximation of conjugate function by de la Vallée-Poussin sums of the Fourier series of the conjugate function in weighted Orlicz spaces $L_{M}(\mathbb{T}, \omega)$. Note that the estimates obtained in this work depend on sequence of the best approximation $E_{n}(f)_{M}, \omega$. Similar problems in different spaces have been investigated by several researchers (see, e.g., $[1,2,11,27,42,43,48-50,52-57,59,60]$ ).

Our main results are as follows.

Theorem 1.7 Let $L_{M}(T)$ be a reflexive Orlicz space and $\omega \in A_{1 / \alpha_{M}}(T) \cap A_{1 / \beta_{M}}(T)$. Then for $f \in$ $L_{M}(T, \omega), 0 \leq m \leq n, m, n \in Z_{+}$the inequality

$$
\left\|f-V_{n, m}(., f)\right\|_{L_{M}(\mathbb{T}, \omega)} \leq \frac{c_{11}}{m+1} \sum_{k=n-m}^{n} E_{k}(f)_{M, \omega}
$$

holds with a constant $c_{11}>0$ independent of $n$.

Note that this result in the spaces of continuous functions and Lebesgue space $L_{p}(1<p<\infty)$ have been investigated in $[49,50,60]$.

Corollary 1.8 Let $L_{M}(T, \omega)$ be a weighted Orlicz space with Boyd indices $0<\alpha_{M} \leq \beta_{M}<1$, and let $\omega \in A_{1 / \alpha_{M}}(T) \cap A_{1 / \beta_{M}}(T), m, n \in Z_{+}, 0 \leq m \leq n$. Then for every $f \in L_{M}(T, \omega)$, the estimate

$$
\left\|f-V_{n, m}(., f)\right\|_{L_{M}(\mathbb{T}, \omega)} \leq \frac{c_{12}}{m+1} \sum_{k=n-m}^{n} \Omega_{M, \omega}^{l}\left(\frac{1}{k+1}, f\right)
$$

holds with a constant $c_{12}>0$ independent of $n$.

Similar result for the other modulus of smoothness in the spaces of continuous functions has been obtained in [49]. Also, similar results for the Cesaro means, Zygmund means of order 2 and Abel-Poisson means in weighted Orlicz spaces can be found in [15].

Theorem 1.9 Let $L_{M}(T, \omega)$ be a weighted Orlicz space with Boyd indices $0<\alpha_{M} \leq \beta_{M}<1$, and let $\omega \in A_{1 / \alpha_{M}}(T) \cap A_{1 / \beta_{M}}(T), m, n \in Z_{+}, 0 \leq m \leq n$. If the inequality

$$
\sum_{\nu=1}^{\infty} \frac{E_{v}(f)_{M, \omega}}{v}<\infty
$$

is satisfied for $f \in L_{M}(T, \omega)$, then the estimate

$$
\left\|\widetilde{f}-V_{n, m}(., \tilde{f})\right\|_{L_{M}(\mathbb{T}, \omega)} \leq c_{13}\left\{\frac{1}{m+1} \sum_{\nu=0}^{n} E_{n-m+v}(f)_{M, \omega .}+\sum_{\nu=n+1}^{\infty} \frac{E_{\nu}(f)_{M, \omega .}}{\nu}\right\}
$$

holds with a constant $c_{13}>0$ independent of $n$.

This result for the spaces of continuous functions has been obtained in [1]. 
Theorem 1.10 Let $\Gamma$ be a Dini-smooth curve. Also, let $L_{M}(\Gamma, \omega)$ be a Orlicz space with Boyd indices $0<$ $\alpha_{M} \leq \beta_{M}<1$, and $\omega \in A_{1 / \alpha_{M}}(\Gamma) \cap A_{1 / \beta_{M}}(\Gamma)$. Then for $f \in E_{M}(G, \omega)$ the inequality

$$
\left\|f-V_{n, m}(., f)\right\|_{L_{M}(T, \omega)} \leq \frac{c_{14}}{m+1} \sum_{k=n-m}^{n} \Omega_{\Gamma, M, \omega}^{l}\left(\frac{1}{k+1}, f\right)
$$

holds with a constant $c_{14}>0$ independent of $n$.

Similar results for the other means of Fourier trigonometric series in the Smirnov classes $E_{p}(G)(1<p<$ $\infty)$ and weighted Orlicz spaces $E_{M}(G, \omega)$ can be found in [15,29].

\section{Proofs of the main results}

Proof of Theorem 1.7 We take the integer $j$ such that the inequality $2^{j} \leq m+1<2^{j+1}$ is satisfied. The following identity holds:

$$
\begin{aligned}
f(x)-V_{n, m}(x, f)= & \frac{1}{m+1}\left[f(x)-S_{n-m}(x, f)\right] \\
& +\frac{1}{m+1}\left\{\sum_{k=1}^{j} \sum_{i=n-m+2^{k-1}}^{n-m+2^{k}-1}\left[f(x)-S_{i}(x, f)\right]\right\} \\
& +\frac{1}{m+1}\left\{\sum_{k=n-m+2^{j}}^{n}\left[f(x)-S_{k}(x, f)\right]\right\} .
\end{aligned}
$$

Taking into account of (2.1), we have

$$
\begin{aligned}
& \left\|f-V_{n, m}(., f)\right\|_{L_{M}(\mathbb{T}, \omega)} \leq \frac{1}{m+1}\left\|f-S_{n-m}(., f)\right\|_{L_{M}(\mathbb{T}, \omega)} \\
& +\frac{1}{m+1}\left\{\sum_{k=1}^{j} \sum_{i=n-m+2^{k-1}}^{n-m+2^{k}-1}\left\|f-S_{i}(., f)\right\|_{L_{M}(\mathbb{T}, \omega)}\right\} \\
& +\frac{1}{m+1}\left\{\sum_{k=n-m+2^{j}}^{n}\left\|f-S_{k}(., f)\right\|_{L_{M}(\mathbb{T}, \omega)}\right\}
\end{aligned}
$$

Consideration of (1.11) and (2.1) gives us

$$
\begin{aligned}
& \left\|f-V_{n, m}(., f)\right\|_{L_{M}(\mathbb{T}, \omega)} \leq c_{15} \frac{1}{m+1}\left\{E_{n-m}(f)_{M, \omega .}+\sum_{k=1}^{j} 2^{k-1} E_{n-m+2^{k-1}}(f)_{M, \omega}\right\} \\
& +c_{15} \frac{1}{m+1}\left(m-2^{j}+1\right) E_{n-m+2^{j}}(f)_{M, \omega} .
\end{aligned}
$$

On the other hand, the following inequality holds:

$$
\begin{aligned}
\sum_{k=1}^{j} 2^{k-1} E_{n-m+2^{k-1}}(f)_{M, \omega} & \leq E_{n-m+1}(f)_{M, \omega} \\
+ & 2 \sum_{k=2}^{j} \sum_{i=n-m+2^{k-2}}^{n-m+2^{k-1}-1} E_{i}(f)_{M, \omega} \leq c_{16} \sum_{k=n-m}^{n-m+2^{j-1}} E_{k}(f)_{M, \omega} .
\end{aligned}
$$


From inequality $2^{j} \leq m+1<2^{j+1}$, we have $2^{j}>m-2^{j}+1$.Then,

$$
\left(m-2^{j}+1\right) E_{n-m+2^{j}}(f)_{M, \omega} \leq \sum_{k=n-m}^{n-m+2^{j}-1} E_{k}(f)_{M, \omega}
$$

Using (2.3), (2.4) and (2.5), we finally conclude that

$$
\begin{aligned}
& \left\|f-V_{n, m}(., f)\right\|_{L_{M}(\mathbb{T}, \omega)} \leq \frac{c_{17}}{m+1}\left\{E_{n-m}(f)_{M, \omega}+\sum_{k=n-m}^{n-m+2^{j-1}} E_{k}(f)_{M, \omega}+\sum_{k=n-m}^{n-m+2^{j}-1} E_{k}(f)_{M, \omega}\right\} \\
& \leq \frac{c_{18}}{m+1} \sum_{k=n-m}^{n} E_{k}(f)_{M, \omega} .
\end{aligned}
$$

Thus, the proof of Theorem 1.7 is completed.

Proof of Corollary 1.8 According to Theorem 1.2 and (1.13), we obtain the inequality (1.14) of Corollary 1.8.

Proof of Theorem 1.9 We consider two cases: 1 . Take $0 \leq 2 m \leq n$. Suppose that $R(x)$ is an antiderivative of the function $f(x)-V_{n, m}(x, f)$ and $U(x)$ is an antiderivative of the function $f(x)-a_{0} / 2$. Then, according to Theorem 1.1 we obtain

$$
E_{v}(U)_{M, \omega} \leq \frac{c_{20}}{v+1} E_{v}(f)_{M, \omega}, \quad v \in Z_{+} .
$$

Since

$$
\sum_{\nu=1}^{\infty} \frac{E_{v}(f)_{M, \omega}}{v}<\infty
$$

the inequality (2.6) yields

$$
\sum_{\nu=1}^{\infty} E_{\nu}(U)_{M, \omega .}<\infty
$$

By virtue of Lemma 1.4 we get

$$
\left\|\widetilde{R}^{\prime}\right\|_{L_{M}(\mathbb{T}, \omega)} \leq c_{21}\left\{n\|R\|_{L_{M}(\mathbb{T}, \omega)}+E_{n}\left(\widetilde{R}^{\prime}\right)_{M, \omega}\right\},
$$

where $R=U-V_{n, m}(., U)+c_{22}$ and $c_{22}$ is a constant. Then, taking Theorem 1.3 into account, we conclude that

$$
E_{n}\left(\widetilde{R}^{\prime}\right)_{M, \omega} \leq c_{23}\left\{(n+1) E_{n}(U)_{M, \omega .}+\sum_{\nu=n+1}^{\infty} E_{\nu}(U)_{M, \omega}\right\} .
$$

Then, the last inequality yields

$$
\left\|\widetilde{f}-V_{n, m}(., \tilde{f})\right\|_{L_{M}(\mathbb{T}, \omega)} \leq c_{24}\left\{(n+1)\left\|f-V_{n, m}(., U)\right\|_{L_{M}(\mathbb{T}, \omega)}+\sum_{\nu=n+1}^{\infty} E_{n}(U)_{M, \omega}\right\} .
$$

From Theorem 1.7 we have

$$
\begin{aligned}
\left\|f-V_{n, m}(., U)\right\|_{L_{M}(\mathbb{T}, \omega)} & \leq \frac{c_{25}}{m+1} \sum_{\nu=n-m}^{n} E_{\nu}(U)_{M, \omega} \\
& \leq \frac{c_{26}}{m+1} \sum_{\nu=n-m}^{n} \frac{E_{\nu}(f)_{M, \omega}}{\nu+1}=\frac{c_{26}}{m+1} \sum_{\nu=0}^{n} \frac{E_{n-m+v}(f)_{M, \omega}}{n-m+v+1} .
\end{aligned}
$$


Since $0 \leq 2 m \leq n$, this implies $(n+1) /(n-m+1) \leq 3$. Then, using the last inequality we reach

$$
\begin{aligned}
& \left\|\widetilde{f}-V_{n, m}(., \tilde{f})\right\|_{L_{M}(\mathbb{T}, \omega)} \leq c_{27}\left\{\frac{1}{m+1} \sum_{\nu=0}^{n} \frac{E_{n-m+v}(f)_{M, \omega}}{n-m+v+1}(n+1)+\sum_{\nu=n+1}^{\infty} E_{\nu}(U)_{M, \omega}\right\} \\
& \leq c_{28}\left\{\frac{1}{m+1} \sum_{\nu=0}^{n} E_{n-m+v}(f)_{M, \omega}+\sum_{\nu=n+1}^{\infty} E_{\nu}(f)_{M, \omega}\right\} .
\end{aligned}
$$

2. Suppose that the inequality $n<2 m \leq 2 n$ is satisfied. From Theorem 1.3 we have

$$
E_{v}(\tilde{f})_{M, \omega} \leq c_{29}\left\{E_{v}(f)_{M, \omega}+\sum_{\mu=v+1}^{\infty} \frac{E_{\mu}(f)_{M, \omega}}{\mu}\right\} .
$$

Using the last inequality and Theorem 1.7 , we find that

$$
\begin{aligned}
& \left\|\widetilde{f}-V_{n, m}(., \tilde{f})\right\|_{L_{M}(\mathbb{T}, \omega)} \leq \frac{c_{30}}{m+1} \sum_{\nu=n-m}^{n} E_{\nu}(\widetilde{f})_{M, \omega} \\
& \leq \frac{c_{31}}{m+1} \sum_{\nu=n-m}^{n}\left\{E_{\nu}(f)_{M, \omega}+\sum_{\mu=\nu+1}^{\infty} \frac{E_{\mu}(f)_{M, \omega}}{\mu}\right\} \\
& \leq c_{32}\left\{\frac{1}{m+1} \sum_{\nu=n-m}^{n} E_{\nu}(f)_{M, \omega}+\sum_{\nu=n+1}^{\infty} \frac{E_{\nu}(f)_{M, \omega}}{\nu}\right\} .
\end{aligned}
$$

Hence, the proof of Theorem 1.9 is completed.

Proof of Theorem 1.10. Suppose that $f \in E_{M}(G, \omega)$. By virtue of Theorem 1.6 the operator $T$ : $E_{M}\left(D, \omega_{0}\right) \longrightarrow E_{M}(G, \omega)$ is bounded, one-to-one and onto and $T\left(f_{0}^{+}\right)=f$. For the function $f$, the following Faber series holds:

$$
f(z) \backsim \sum_{k=0}^{\infty} a_{k}(f) \varphi_{k}(z)
$$

Since $\omega_{0} \in A_{1 / \alpha_{M}}(T) \cap A_{1 / \beta_{M}}(T)$, considering Lemma 1.4 given in Ref. [20] we get $f_{0}^{+} \in E_{M}\left(D, \omega_{0}\right)$. Then, function $f_{0}^{+}$has the following Taylor expansion

$$
f_{0}^{+}(w)=\sum_{k=0}^{\infty} a_{k}(f) w^{k} .
$$

Note that $f_{0}^{+} \in E_{1}(D)$ and boundary function $f_{0}^{+} \in L_{M}\left(T, \omega_{0}\right)$. Then, using Theorem 3.4 [8, p.38] for the function $f_{0}^{+}(w)$ we get Fourier expansion

$$
f_{0}^{+}(t) \backsim \sum_{k=0}^{\infty} a_{k}(f) e^{i t k} .
$$

Using the boundedness of the operator $T$, Theorems 1.7 and 1.2 we reach

$$
\begin{aligned}
& \left\|f-V_{n, m}(., f)\right\|_{L_{M}(\Gamma, \omega)}=\left\|T\left(f_{0}^{+}\right)-T\left(V_{n, m}\left(., f_{0}^{+}\right)\right)\right\|_{L_{M}(\Gamma, \omega)} \\
& \leq c_{33}\left\|f_{0}^{+}-V_{n, m}\left(., f_{0}^{+}\right)\right\|_{L_{M}\left(T, \omega_{0}\right)} \leq \frac{c_{34}}{m+1} \sum_{k=n-m}^{n} E_{k}\left(f_{0}^{+}\right)_{M, \omega} \\
& \leq \frac{c_{35}}{m+1} \sum_{k=n-m}^{n} \Omega_{M, \omega_{0}}^{l}\left(\frac{1}{k+1}, f_{0}^{+}\right)=\frac{c_{35}}{m+1} \sum_{k=n-m}^{n} \Omega_{\Gamma, M, \omega}^{l}\left(\frac{1}{k+1}, f\right) .
\end{aligned}
$$


Thus, the theorem is proved.

Acknowledgments The author would like to thank the referees for their helpful remarks and corrections.

Open Access This article is distributed under the terms of the Creative Commons Attribution 4.0 International License (http:// creativecommons.org/licenses/by/4.0/), which permits unrestricted use, distribution, and reproduction in any medium, provided you give appropriate credit to the original author(s) and the source, provide a link to the Creative Commons license, and indicate if changes were made.

\section{References}

1. Baiborodov, S.P.: Approximation of functions by Vallé e-Poussin sums. Matem. Zametki, 24(1), 33-47 (1980) (in Russian)

2. Baiborodov, S.P.: Approximation of conjugate functions by Fourier series sums in $L_{2 \pi}^{p}$. Anal. Math. 11, 3-12 (1985)

3. Bennett, C., Sharpley, R.: Interpolation of Operators. Academic Press, New York (1988)

4. Böttcher, A.; Karlovich, Y.I.: Carleson Curves, Muckenhoupt Weights and Teoplitz Operators. Birkhauser, Verlag (1997)

5. Boyd, D.W.: Spaces between a pair of reflexive Lebesgue spaces. Proc. Am. Math. Soc. 18, 215-219 (1967)

6. Boyd, D.W.: Indices of function spaces and their relationship to interpolation. Can. J. Math. 21, 1245-1254 (1969)

7. Boyd, D.W.: Indices for the orlicz spaces. Pacific J. Math. 38, 315-325 (1971)

8. Duren, P.L.: Theory of $H^{p}$ Spaces. Academic Press, New York (1970)

9. Ditzian, Z., Totik, V.: Moduli of Smoothness. Springer Ser, Copmut. Math. 9, Springer, New York (1987)

10. Devore, R.A., Lorentz, G.G.: Constructive approximation. Springer, New York (1993)

11. Gavriljuk, V.T.: Linear summability methods for Fourier series and best approximation. Ukrain. Math. Zh. 15(4), 412-418 (1963) (in Russian)

12. Goluzin, G.M.: Geometric Theory of Functions of a Complex Variable. Translation of Mathematical Monographs, vol. 26, Providence, RI: AMS, 1968

13. Gadjieva, E.A.: Investigation of the properties of functions with quasimonotone Fourier coefficients in generalized NikolskiiBesov spaces. Author's summary of dissertation, Tbilisi, 1986 (in Russian)

14. Guven, A.: Trigonometric approximation of functions in weighted $L^{p}$ spaces. Sarajevo J. Math. 5(17), 99-108 (2009)

15. Guven, A., Israfilov, D.M.: Approximation by Means of Fourier trigonometric series in weighted Orlicz spaces. Adv. Stud. Contemp. Math. (Kyundshang), 19(2), 283-295 (2009)

16. Ibragimov, I.I., Mamedkhanov, D.I.: A constructive characterization of a certain class of functions. Dokl. Akad. Nauk. SSSR 223(1), 35-37 (1975) (in Russian)

17. Israfilov, D.M., Approximation by p-Faber polynomials in the weighted Smirnov class $E^{p}(G, \omega)$ and the Bieberbach polynomials. Constr. Approx. 17(3), 335-351 (2001)

18. Israfilov, D.M., Guven, A.: Approximation by trigonometric polynomials in weighted Orlicz spaces. Stud. Math. 174(2), $147-168$ (2006)

19. Israfilov, D.M., Oktay, B., Akgün, R.: Approximation in Smirnov-Orlicz classes. Glas. Mat. Ser. III 40(60), 87-102 (2005)

20. Israfilov, D.M., Akgün, R.: Approximation in weighted Smirnov-Orlicz classes. J. Math. Kyoto Univ. 46(4), 755-770 (2006)

21. Israfilov, D.M., Kokilashvili, V.M., Samko, S.G.: Approximation in weighted Lebesgue and Smirnov spaces with variable exponent. Proc. A. Razmadze Math. Inst. 143, 25-35 (2007)

22. Jafarov, S.Z.: Approximation by rational functions in Smirnov-Orlicz classes. J. Math. Anal. Appl. 379, $870-877$ (2011)

23. Jafarov, S.Z.: Approximation of functions by rational functions on closed curves of the complex plane. Arab. J. Sci. Eng. 36(8), 1529-1531 (2011)

24. Jafarov, S.Z.: On approximation in weighted Smirnov-Orlicz classes. Complex Var. Elliptic Equ. 57(5), 567-577 (2012)

25. Jafarov, S.Z.: The inverse theorem of approximation of the function in Smirnov-Orlicz classes. Math. Ineq. Appl. 12(4), 835-844 (2012)

26. Jafarov, S.Z.: Approximation of conjugate functions by trigonometric polynomials in weighted Orlicz spaces. J. Math. Ineq. 7(2), 271-281 (2013)

27. Jafarov, S.Z.: Approximation by Fejér sums of Fourier trigonometric series in weighted Orlicz spaces. Hacet. J. Math. Stat. 42(3), 259-268 (2013)

28. Krasnoselskii, M.A., Rutickii, Y.B.: Convex Functions and Orlicz Spaces. P. Norrdhoff Ltd., Groningen (1961)

29. Kokilasvili, V.M.: On approximation of analytic functions from $E_{p}$ classes. Trudy Tbiliss. Mat. Inst. im Razmadze Akad. Nauk Gruzin. SSR 34, 82-102 (1968)(in Russian)

30. Kokilashvili, V.M.: On analytic functions of Smirnov-Orlicz classes. Stud. Math. 31, 43-59 (1968)

31. Kokilashvili, V.M., Samko, S.G.: Operators of harmonic analysis in weighted spaces with non-standard growth. J. Math. Anal. Appl. 352, 15-34 (2009)

32. Kokilashvili, V.M., Samko, S.G.: A refined inverse inequality of approximation in weighted variable exponent Lebesgue spaces. Proc. A. Razmadze Matgh. Inst. 151, 132-138 (2009)

33. Karlovich, A.Y.: Algebras of Singular integral operators with piecewise continuous coefficients on reflexive Orlicz spaces. Math. Nachr. 179, 187-222 (1996)

34. Karlovich, A.Y.: Singular integral operators with PC coefficients in reflexive rearrangement invariant spaces. Integr. Eq. Oper. Theory 32, 436-481 (1998)

35. Karlovich, A.Y.: Fredholmness of singular integral operators with piecewise continuous coefficients on weighted Banach function spaces. J. Integr. Eq. Appl. 15, 263-320 (2003)

36. Ky, N.X.: On approximation by trigonometric polynomials in $\mathrm{L}_{u}^{p}$-spaces. Stud. Sci. Math. Hungar. 28, $183-188$ (1993)

37. Ky, N.X.: Moduli of mean smoothness and approximation with $A_{p}$ weights. Ann. Univ. Sci. Budapest 40, 37-48 (1997) 
38. Matuszewska, W., Orlicz, W.: On certain properties of $\phi$-functions. Bull. Acad. Polon. Sci. Ser. Math. Aster. Phys. 8(7), 439-443 (1960)

39. Maligranda, L.: Indices and interpolation. Dissertationes Math. vol. 234 (1985)

40. Mamedkhanov, D.I.: Approximation in complex plane and singular operators with a Cauchy kernel. Dissertation Doct. Phys-math.nauk. The University of Tblisi, 1984 (in Russian)

41. Mhaskar, H.N.: Introduction to the theory of weighted polynomial approximation. Series in Approximation and Decompositions 7, World Sci., River Edge, NJ (1996)

42. Ovsi, Y., Serdyuk, A.S.: Approximation of continuous periodic functions by de la Vallée Poussin sums. Zb. Pr. Inst. Mat. NAN Ukr. 8(1), 151-161 (2011)

43. Prestin, J.: On the approximation by de la Vallée Poussin sums and interpolatory polynomials in Lipschitz norms. Anal. Math. 14, 251-259 (1987)

44. Pommerenke, C.: Boundary Behavior of Conformal Maps. Springer, Berlin (1992)

45. Ponomarenko, V.G.: Approximation of periodic functions in a Orlicz space. Sibirsk. Math. J. 7, 1337-1346 (1966) (in Russian)

46. Ramazanov, A.R.K.: On approximation by polynomials and rational functions in Orlicz spaces. Anal. Math. 10, 117-132 (1984)

47. Rao, M.M., Ren, Z.D.: Theory of Orlicz Spaces. Marcel Dekker, New York (1991)

48. Stechkin, S.B.: On best approximation of conjugate functions by trigonometric polynomials. Izv. Akad. Nauk SSSR Ser. Mat. 20(2), 197-206 (1956) (in Russian)

49. Stechkin, S.B.: The approximation of periodic functions by Fejér sums. Trudy Math Inst. Steklov 62, 48-60 (1961)(in Russian)

50. Stechkin, S.B.: On the approximation of periodic functions by de la ValléePoussin sums. Anal. Math. 4, 61-74 (1978)

51. Suetin, P.K.: Series of Faber Polynomials, vol. 1. Gordob and Breack, Reading (1998)

52. Serdyuk, A.S., Ovsi, Y., Musienko, A.P.: Approximation of classes of analytic functions by de la ValléePoussin sums in uniform metric. Rend. Mat. Appl. 32, 1-15 (2012)

53. Timan, A.F.: Theory of Approximation of Functions of a Real Variable. Pergamon Press, MacMillan (1963) Russian original published by Fizmatgiz, Moscow, 1960.

54. Timan, M.F.: Best approximation of a function and linear methods of summing Fourier series. Izv. Akad. Nauk SSSR Ser: Math. 29, 587-604 (1965)(in Russian)

55. Timan, M.F.: The approximation of continuous periodic functions by linear operators which are constructed on the basis of their Fourier. Ser. Dokl. Akad. Nauk SSSR 181, 1339-1342 (1968)(in Russian)

56. Timan, M.F.: Some linear summation processes for Fourier series and best approximation. Dokl. Akad. Nauk SSSR 145, 741-743 (1962)

57. Vallée Poussin, C.L.: Sur la meilleure approximation des fonctions d'une variable reelle par des expressions d'ordre donné. Comptes dendus de l'Académie des Sciences 166(4), 799-802 (1918)

58. Warschawskii, S.: Über das Randverhalten der Ableitung der Abbildungsfunktionen bei Konformer Abbildung. Math. Z. 35, 321-456 (1932)

59. Zhizhiashvili, L.V.: Trigonometric Fourier series and their conjugates, Tbilis. Gos. Univ. Tbilisi, 1993 (in Russian); English transl.: Kluwer Acad. Publ., Dordrecht, 1996.

60. Zakharov, A.A.: Bound on deviations of continuous periodic functions from their De La Vallée-Poussin sums. Matem. Zametki, 3(1), 77-84 (1968)(in Russian) 